\title{
Applications of Steroid in Clinical Practice: A Review
}

\author{
Safiya Shaikh, ${ }^{1}$ Himanshu Verma, ${ }^{2}$ Nirmal Yadav, ${ }^{3}$ \\ Mirinda Jauhari, ${ }^{4}$ and Jyothi Bullangowda ${ }^{5}$ \\ ${ }^{1}$ Department of Anaesthesiology, Karnataka Institute of Medical Sciences (KIMS), Hubli 580029, India \\ ${ }^{2}$ Department of Anaesthesiology, SRMS IMS, Bhojipura, Bareilly 243202, India \\ ${ }^{3}$ Department of Internal Medicine, SRMS IMS, Bhojipura, Bareilly 243202, India \\ ${ }^{4}$ Department of Pulmonary Medicine, SRMS IMS, Bhojipura, Bareilly 243202, India \\ ${ }^{5}$ Department of Anaesthesiology, KIMS, Hubli 580029, India
}

Correspondence should be addressed to Safiya Shaikh, ssafiya11@yahoo.com and Jyothi Bullangowda, dr.jyothib1@gmail.com

Received 5 August 2012; Accepted 27 August 2012

Academic Editors: D. Karakitsos and F. Khan

Copyright (c) 2012 Safiya Shaikh et al. This is an open access article distributed under the Creative Commons Attribution License, which permits unrestricted use, distribution, and reproduction in any medium, provided the original work is properly cited.

\begin{abstract}
Steroids are perhaps one of the most widely used group of drugs in present day anaesthetic practice, sometimes with indication and sometimes without indications. Because of their diverse effects on various systems of the body, there has been renewed interest in the use of steroids in modern day anaesthetic practice. This paper focuses on the synthesis and functions of steroids and risks associated with their supplementation. This paper also highlights the recent trends, relevance, and consensus issues on the use of steroids as adjunct pharmacological agents in relation to anaesthetic practice and intensive care, along with emphasis on important clinical aspects of perioperative usefulness and supplementation.
\end{abstract}

\section{Introduction}

Corticosteroids and their biologically active synthetic derivatives differ in their metabolic (glucocorticoid) and electrolyte-regulating (mineralocorticoid) activities. These agents are employed at physiological doses for replacement therapy when endogenous production is impaired. In addition, glucocorticoids potently suppress inflammation, and their use in a variety of inflammatory and autoimmune diseases makes them among the most frequently prescribed classes of drugs $[1,2]$.

The effects of corticosteroids are numerous and widespread and include alterations in carbohydrate, protein lipid metabolism; maintenance of fluid and electrolyte balance; and preservation of normal function of the cardiovascular system, the immune system, the kidney, skeletal muscle, the endocrine system, and the nervous system. In addition, corticosteroids endow the organism with the capacity to resist such stressful circumstances as noxious stimuli and environmental changes $[1,3,4]$.

\section{Regulation of Cortisol Secretion}

The following three major mechanisms control ACTH release and the Cortisol secretion.

(a) Negative feedback mechanism: the most important stimulus for secretion of cortisol is the release of ACTH from anterior pituitary. The secretion of ACTH in anterior pituitary is determined by two hypothalamic neurohormones (diurnal release of CRF and AVP) that act synergistically [3-5]. Circulating cortisol also exerts a direct negative feedback on the hypothalamus and anterior pituitary to decrease the release of CRF and ACTH from respective sites.

(b) Diurnal variation: cortisol is secreted from adrenal gland in an episodic manner and frequency of pulses follows a circadian rhythm that is dependent on both day-night and sleep-wake patterns and is disrupted by alternating day-night shift working patterns and by long distance travel across time zones. It may take 
up to 2 weeks for circadian rhythm to reset to an altered day-night cycle. Levels are the highest in the morning on waking and the lowest in the middle of evening. Maximal cortisol level occurs at 4-6 am and is lower at 4-8 pm [3-5].

(c) Stress: Stress such as physical (trauma, surgery, exercise); psychological (pain, anxiety, apprehension); physiological (nausea, fever and hypoglycemia) can over ride the negative feedback mechanism and diurnal variation. Cortisol rises immediately (within minutes) and dramatically during stress. This elevation protects key metabolic functions and puts an important "brake" on potentially damaging inflammatory responses to infection and injury [6].

\section{Glucocorticoids: Physiological Effect}

3.1. Metabolism. The principal glucocorticoid cortisol is an essential regulator of carbohydrate, protein, lipid, and nucleic acid metabolism.

Cortisol stimulates gluconeogenesis by liver resulting in increased blood glucose concentrations [3, 4, 7]. One of the principal effects of cortisol on the metabolic system of body is reduction of protein stores essential in all body cells, except those of liver. This is caused by both decreased protein synthesis and increased catabolism of proteins already in cells $[1,3,4]$. Cortisol promotes mobilization of fatty acids from adipose tissue and enhances oxidation of fatty acids in cells; excess cortisol causes deposition of fat in neck and chest regions, giving a "buffalo like" torso $[1,3,4]$.

3.2. Anti-Inflammatory Effects. Cortisol in large amounts has anti-inflammatory effects, reflecting its ability to stabilize lysozyme membranes, decrease the release of inflammation causing lysozymes, and decrease capillary permeability which prevents loss of plasma protein in to tissues [3-5, 7].

It may also interfere with complement pathway activation and formation of chemical mediator derived from arachidonic acids such as leukotrienes [7].

3.3. Bone and Calcium Metabolism. Osteoblast function is inhibited by glucocorticoids and this is thought to be the explanation for osteopenia and osteoporosis that characterize glucocorticoid excess $[3,4]$.

3.4. Blood Pressure Control. Glucocorticoids increase blood pressure by a variety of mechanisms involving actions on the kidney and vasculature. In the vascular smooth muscle they increase sensitivity to pressor agents such as catecholamines and angiotensin II, while reducing nitric oxide-mediated endothelial dilatation [3-5].

3.5. CNS and Mood. Clinical observation of patients with glucocorticoid excess and deficiency reveals that brain is an important target for glucocorticoids with depression, euphoria, psychosis, apathy, and lethargy being important manifestations $[3,4]$.
3.6. Developmental Changes. Plasma cortisol in last trimester of pregnancy is necessary for maturing of number of systems that are critical for survival of fetus in extrauterine life. These systems include production of pulmonary surfactant, maturation of various enzyme systems in the liver, and the expression of phenylethanolamine $\mathrm{N}$-methyltransferase, the enzyme necessary for synthesis of epinephrine from norepinephrine $[3,4]$.

3.7. Eye. In the eye, glucocorticoids act to raise intraocular pressure through an increase in aqueous humor production and deposition of matrix within trabecular meshwork which inhibits aqueous drainage $[3,4]$.

3.8. Gut. Chronic but not acute administration of glucocorticoid increases the risk of developing peptic ulcer disease. Pancreatitis with fat necrosis is reported in patients with glucocorticoid excess $[3,4,7]$.

3.9. Endocrine Effects. Glucocorticoids suppress thyroid axis, probably through a direct action on thyroid stimulating hormone secretion. It acts centrally to inhibit gonodotrophinreleasing hormone pulsatality and release of lutenizing hormone and follicular stimulating hormone.

\section{Effect of Anaesthesia and Surgery}

Plasma cortisol levels typically increase from two-to-tenfolds following induction of anesthesia, during surgery, and in postoperative period. The maximum ACTH and cortisol levels are reached in the early postoperative period, especially following anaesthesia reversal and endotracheal extubation (30 min after extubation) [8-10].

As with other types of stress, the episodic release of cortisol remains intact, but the amplitude of this episodic release is increased. The increase in the plasma cortisol concentration may in part be due to bar receptors and spinal reflexes that signal the hypothalamus of tissue injury associated with surgery $[3,4]$.

Other factors that activate HPA axis to release cortisol include proinflammatory mediators released by damaged tissues and presence of postoperative pain.

Plasma cortisol concentrations typically return to normal levels within 24 hrs postoperatively but may remain elevated as long as $72 \mathrm{hrs}$, depending on severity of the surgical trauma.

Return of the plasma concentration to normal following surgery is initially characterized by increased plasma concentration of ACTH and cortisol (in the first 24hrs) $[11,12]$ followed by a second phase $(48-72 \mathrm{hrs})[10,13]$ in which plasma ACTH concentrations are low and increased plasma cortisol concentrations are presumably independent of HP system [14].

In addition to surgical trauma, choice of anesthetic drugs and techniques may influence the HPA response. For example, large doses of opioid may attenuate the cortisol response to surgical stimulation [15]. Volatile anesthetics provide less suppression to this stress-induced endocrine response 
TABLE 1: Classification and comparison of systemic steroids $[1,7]$.

\begin{tabular}{lcccc}
\hline Glucocorticoid & Anti-inflammatory potency & Na-retaining potency & Duration of action & Equivalent dose \\
\hline Cortisol & 1 & 1 & Short $(<12 \mathrm{hrs})$ & \\
Cortisone & 0.8 & 0.8 & \\
Prednisone & 4 & 0.8 & & \\
Prednisolone (P) & 4 & 0.8 & Intermediate $(12-36 \mathrm{hrs})$ & 5 \\
6 methyl P & 5 & 0.5 & & 5 \\
Triamcinolone & 5 & 0 & Large $(>36 \mathrm{hrs})$ & 4 \\
Dexamethasone & 25 & 0 & & 0.75 \\
Betamethasone & 25 & 0 & & 0.75
\end{tabular}

[15]. Etomidate is unique among drugs administered to induce anesthesia with respect to its ability to inhibit cortisol synthesis (selectively inhibits adrenal 11 beta hydroxylase, the enzyme that converts 11 deoxy cortisol to cortisol) even in the absence of surgical stimulation [16].

Some medications other than glucocorticoids may suppress HPA function and place patients at the risk of developing adrenal insufficiency. Progestational agents such as medroxyprogesterone and megestrol have glucocorticoid activity. Enzyme inducers such as rifampin and carbamazepine enhance the clearance of some synthetic glucocorticoids [17]. Inhibitors of cortisol synthesis include ketoconazole, aminoglutethimide, and etomidate [16].

Previous studies have shown that cortisol and ACTH levels increase during normal pregnancy, particularly in the second and third trimesters [18]. Some authorities have recommended increasing glucocorticoid replacement doses by $50 \%$ in the last trimester of pregnancy for women with adrenal insufficiency. Whether this is advisable may depend on the patients usual treatment dose, as it has been shown that a dose increase is rarely necessary in women treated with 20-30 mg hydrocortisone daily.

Glucocorticoids are classified according to the duration of action. Short acting ones include cortisol and cortisone with duration of action less than $12 \mathrm{hrs}$ and antiinflammatory and sodium retaining potency of 1 and 0.8 , respectively. Intermediate acting ones (duration of action 12-36 hrs) include prednisolone and prednisone with antiinflammatory and sodium retaining potency of 4 and 0.8 , 6-methyl prednisolone with anti-inflammatory and sodium retaining potency of 5 and 0.5 , and triamcinolone with antiinflammatory and sodium retaining potency of 5 and 0 . Long acting ones include dexamethasone and beclomethasone with duration of action greater than $36 \mathrm{hrs}$ and antiinflammatory and sodium retaining potency of 25 and 0 (Table 1).

\section{Important Indications of Steroid in Anaesthetic Practice}

5.1. Perioperative Steroid Replacement Therapy. Corticosteroid supplementation should be provided for patients being treated with steroids either for hypocortisolism or for other diseases. This is based on the concern that these patients are more prone to cardiovascular collapse as release of additional endogenous cortisol in response to surgical stress is not likely. Some patients may display suppression of pituitary-adrenal axis with atrophy of adrenal cortex from long continued therapy with steroid drugs.

Steroid administration is necessary in perioperative period in patients treated for hypoadrenocorticism or in patients with suppression of HPA axis owing to previous or present steroid intake [19]. The increase in circulating cortisone levels from normal of $25 \mathrm{mg} /$ day to up to $300 \mathrm{mg}$ /day in severe surgical stress is one of the important components of stress response of our body. In the perioperative period due to adrenal suppression, there can be increased vascular permeability, inadequate vasomotor response, decrease in cardiac output, and decrease in systemic vascular resistance and left ventricular stroke volume index which can lead to severe hypotension and cardiovascular collapse, respiratory depression, hyponatremia, hypoglycemia, hypercalcemia, and hemoconcentration [20].

The specific duration and dose of steroid that can produce HPA suppression is controversial. The recovery time of normal HPA axis varies from 2-5 days to 9-12 months after discontinuation of steroid therapy. But the ability to respond to stress returns by 2 months [21].

Traditionally it was believed that the degree of HPA suppression and adrenal atrophy in patients receiving exogenous glucocorticoids was related to duration and dose of therapy $[22,23]$. In patients taking steroids for less than 3 weeks, suppression of HPA axis is rarely clinically insignificant [23]. Conversely, any patient who has received the equivalent of $15 \mathrm{mg} /$ day of prednisolone for more than 3 weeks should be suspected of having HPA suppression [23]. However recent studies have found poor correlation between HPA axis function and the cumulative dose or the duration of therapy $[24,25]$. Because of considerable interindividual variability in the degree and duration of adrenal suppression, it is difficult to accurately predict which patients will develop adrenal insufficiency when glucocorticoid treatment is discontinued. Thus the need to evaluate HPA is a frequent consideration.

Under perioperative conditions adrenal glands secrete $116-185 \mathrm{mg}$ of cortisol daily. If plasma cortisol is measured during acute stress, a value of more than $25 \mu \mathrm{g} / \mathrm{dL}$ assuredly and more than $15 \mu \mathrm{g} / \mathrm{dL}$ probably indicates normal pituitaryadrenal responsiveness [26].

The intactness of the HPA axis and need for steroid may be assessed by provocative tests which measure the plasma cortisol response to administration of $\mathrm{ACTH}, \mathrm{CRH}$, lysine, 
vasopressin, metyrapone, and insulin-induced hypoglycemia [26]. The gold standard for assessment of HPA function is the insulin tolerance test, but short synacthen test is cheaper and less unpleasant [27].

\subsection{Tests for Adequacy of HPA Axis}

(1) IV Regular Insulin $0.1-0.15 \mathrm{U} / \mathrm{kg}$ results in rapid lowering of blood sugar level within 10-20 min to less than $2.2 \mathrm{mmol} / \mathrm{lt}$ which triggers the release of ACTH from pituitary and cortisol from adrenal cortex. This indicates the adequacy of HPA axis function $[1,5]$.

(2) $30 \mathrm{Min}$ ACTH Test is the most consistent and accurate diagnostic tool for preoperative evaluation of HPA axis function. Synthetic ACTH (cosyntropin) in a dose of $250 \mu \mathrm{g}$ is administered IV and a blood sample for plasma cortisol is collected 30 mins later. Plasma cortisol concentration more than $500 \mathrm{nmol} / \mathrm{lt}$ $(18-20 \mu \mathrm{g} / \mathrm{dL})$ defines adequate adrenal function. This test is recommended as a preoperative screening test for evaluation of HPA integrity $[1,5]$.

(3) Short Synatchen Test: $250 \mu \mathrm{g}$ of ACTH is given and cortisol is disease measured at 0 and 30 mins. Addison's is excluded if second cortisol is $>500 \mathrm{nmol} / \mathrm{L}$ and $>200 \mathrm{nmol} / \mathrm{L}$ greater than baseline. If this does not exclude Addison's, an ACTH level should be measured (low cortisol and ACTH $>300$, Addison's) $[1,5]$.

Original recommendations for perioperative steroid supplementation were in excess of what was actually required. Based on Udelsman et al., it was concluded that there is no advantage in supraphysiologic glucocorticoid prophylaxis during surgical stress, and replacement doses of cortisol equivalent to the daily unstressed cortisol production rate are sufficient to allow homeostatic mechanisms to function during surgery [28].

The present approach is to replace the amount equivalent to normal physiologic response to surgical stimuli. The amount of steroid supplementation, dose, and duration should be based on the magnitude of surgical stress as well as perioperative steroid dose and degree of HPA suppression. Also it is important to note that oral steroids must be supplemented by parenteral steroids in equivalent doses [29].

Salem et al., [30] categorized the following:

(a) minor surgical stress as inguinal herniorrhaphy, appendicectomy, dental procedures of $>1 \mathrm{hr}$ duration under local anaesthesia, and caesarean section [30];

(b) moderate surgical stress as non laparoscopic cholecystectomy, abdominal hysterectomies, lower limb revascularisation, segmental colon resection, THR [30];

(c) severe surgical stress as Whipple's resection, esophagectomy, pituitary adrenalectomy, total proctocolectomy, liver resection, cardiopulmonary bypass, dental procedures under general anaesthesia, orthognathic surgery, and severe facial trauma [30].
One of the widely practised steroid replacements in perioperative setting is given by Salem et al. [30].

Patients taking steroids $<10 \mathrm{mg} /$ day (prednisolone) are assumed to have normal HPA response and they do not need any steroid cover. Patients taking steroids $>10 \mathrm{mg} /$ day (prednisolone) and posted for minor surgery should be given $25 \mathrm{mg}$ hydrocortisone at induction. Patients taking steroids $>10 \mathrm{mg} /$ day (prednisolone) and posted for moderate surgery should be administered usual perioperative steroid +25 hydrocortisone at induction $+100 \mathrm{mg} /$ day for $24 \mathrm{hrs}$. Patients taking steroids $>10 \mathrm{mg} /$ day (prednisolone) and posted for major surgery should be administered usual perioperative steroid +25 hydrocortisone at induction + $100 \mathrm{mg} /$ day for $48-72 \mathrm{hrs}$. Patients on high dose immunosuppressive steroids should be continued on usual immunosuppressive dose during perioperative period. Patients who have stopped taking steroids within last 3 months should be considered as patients on steroids and supplementation should be made accordingly. Patients who have stopped steroids more than 3 months back do not require any perioperative steroid supplementation (Table 2).

Alternately, another regimen is to give $100 \mathrm{mg}$ hydrocortisone followed by, in minor cases $100 \mathrm{mg}$ 6-8 hrs for $24 \mathrm{hrs}$ and in major cases $100 \mathrm{mg} \mathrm{6-8} \mathrm{hrs} \mathrm{for} 72 \mathrm{hrs} \mathrm{[31].}$

\subsection{Steroid Coverage for Illness or Surgery in Hospital [32]}

(a) For minimal illness (non febrile cough or upper respiratory tract infection), usual replacement dose should be given $[32,33]$.

(b) For minor illness (viral illness, bronchitis, and uncomplicated urinary tract infection), double or triple the usual dose of glucocorticoid should be again until recovery $[32,33]$.

(c) For moderate illness (gastroenteritis, pneumonia, pyelonephritis) give hydrocortisone $50 \mathrm{mg}$ bd orally or iv. Taper rapidly to maintenance dose as patient recovers $[32,33]$.

(d) For severe illness (pancreatitis, myocardial infarction, labour) give hydrocortisone $100 \mathrm{mg}$ iv every 8 th hrly. Taper dose to maintinence level by decreasing by half every day. Adjust dose according to course of illness $[32,33]$.

(e) For minor procedures under LA and most radiological studies, no extra supplementation is needed [32].

(f) For moderately stressful procedures, such as barium enema, endoscopy, or arteriography, give a single $100 \mathrm{mg}$ iv dose of hydrocortisone just before the procedure [32].

(g) For major surgery, give hydrocortisone $100 \mathrm{mg}$ iv just before induction of anaesthesia and continue every $8 \mathrm{hrs}$ for the first $24 \mathrm{hrs}$. Taper dose rapidly to maintenance level by decreasing by half every day [32].

5.3.1. Patients with HPA Axis Depression. Based on Kehlet and Binder [33], in patients with proven adrenocortical 





insufficiency, a low dose physiological substitution regimen results in circulating cortisol values greater than in normal patients and is sufficient to prevent intraoperative hemodynamic instability. An infusion is preferable as it avoids large increase caused by bolus doses [33]. A rational regimen for steroid supplementation in the perioperative period is administration of cortisol $25 \mathrm{mg}$ iv, at the induction of anaesthesia followed by continuous infusion of cortisol $100 \mathrm{mg}$ during the following $24 \mathrm{hrs}$. This approach maintains the plasma concentration of cortisol above normal during major surgery in patients receiving chronic treatment with steroids and manifesting a subnormal response to preoperative infusion of ACTH [33]. In addition to intravenous supplementation with cortisol, patients receiving daily maintenance doses of steroids should also receive this dose with premedication on the day of surgery. In those instances like burns or sepsis which exaggerate the need for exogenous steroid supplementation, the continuous infusion of cortisol, $100 \mathrm{mg}$ every $12 \mathrm{hrs}$ is sufficient [33].

\section{Application of Steroids in PONV}

The antiemetic mechanism of corticosteroids is unknown [34]. Dexamethasone, a corticosteroid with strong antiinflammatory effects, provides postoperative analgesia [35], prevents nausea and vomiting in patients undergoing chemotherapy [36], and reduces postoperative nausea and vomiting (PONV) [37]. Dexamethasone may inhibit the synthesis of prostaglandin and various other inflammatory mediators, which are known to act on chemoreceptor trigger zone and cause emesis [38]. Previous studies suggested that decreased serotonin release in the central nervous system and changes in the permeability of the blood cerebrospinal fluid barrier to serum proteins [39] may also play a role in the antiemetic effects of corticosteroids.

\section{Use of Steroids in Ambulatory Surgery}

Postoperative and postdischarge nausea and vomiting (PONV/PDNV) are the most common complications of ambulatory surgery [40]. Gupta et al. [41] in a meta-analysis review, showed the incidence of postoperative Vomiting (0$55 \%)$ and postdischarge Vomiting (0-16\%). Patients with PONV are significantly more likely to have problems performing activities of daily life have a lower satisfaction score and higher negative economic impact than those not experiencing PONV.

Steroids decrease the incidence of PONV, postoperative pain, establish early oral intake, stimulate appetite, and induce a sense of well being (due to increase in release of endorphins). Dexamethasone is a corticosteroid with potent anti-inflammatory effects that contribute to decreased wound pain after oral surgery [42]. It also has antiemetic properties in patients receiving highly emetogenic chemotherapy [43].

More recently, dexamethasone has been used as a prophylactic antiemetic in the ambulatory surgery setting [44]. Aesboe et al. conducted a study on use of intramuscular single dose $12 \mathrm{mg}$ betamethasone, $30 \mathrm{~min}$ before ambulatory hemorrhoidectomy or hallux valgus correction, and they found significantly less PONV, less post operative pain, and better patient satisfaction [44].

\section{Steroids and There Applications as Analgesic Adjuncts}

Analgesic effect of steroid is suspected to be mediated by anti-inflammatory and immune suppressive effect [45]. Its anti-inflammatory action results in decreased production of various inflammatory mediators that play a major role in amplifying and maintenance of pain perception. Some studies have demonstrated the analgesic effect of local spinal and systemic corticosteroids in combination with bupivacaine [46]. Dexamethasone microspheres have been found to prolong the block duration in animal and human studies, and adding methylprednisolone to local anesthetic increases the duration of axillary brachial block.

Movafegh et al. [47] compared addition of $8 \mathrm{mg}$ dexamethasone to $34 \mathrm{~mL}$ of $1.5 \%$ lidocaine to that of $1.5 \%$ of lidocaine. They concluded that the duration of sensory $(242 \pm 76 \mathrm{~min}$ in dexamethasone group versus $98 \pm 33 \mathrm{~min}$ in control group $)$ and motor $(310 \pm 817 \mathrm{~min}$ in dexamethasone group versus $130 \pm 31 \mathrm{~min}$ in control group) blockade was significantly longer in the dexamethasone than in the control group [47].

Paracetamol, NSAIDs, and glucocorticoids have a ceiling of analgesic effect, not being sufficient as monotherapy after extensive surgery. As glucocorticoids act on the prostaglandin system differently than NSAIDs and have other antiinflammatory effects, there may be better analgesia when glucocorticoids are added to NSAIDs.

Adverse effects with a single dose of dexamethasone are probably extremely rare and minor in nature, and previous studies have demonstrated that short-term $(<24$ hours $)$ use of dexamethasone was safe [48].

\section{Epidural Steroid Injection (ESI)}

ESI have been used to treat back pain (mainly due to nerve root irritation) in patients with a wide variety of spine pathologies including radiculopathy, spinal stenosis, diskspace narrowing, annular tears, spondylosis, spondylolisthesis, vertebral fractures, and postlaminectomy syndrome [49]. ESI provides an effective alternative to surgical treatment and is best for patients with lumbar disc disease who have not improved after 4 weeks of conservative medical therapy.

The purpose of an ESI is to deliver medication directly to the affected nerve roots, thereby limiting the effects of systemically administered steroids. Difference of opinion exists as to what represents the optimal treatment or what constitutes a "proper ESI." It extends to virtually all aspects of ESI including the type, dose, volume of steroid injectate, frequency of administration, approaches, and methods of identifying epidural space.

A common regimen is use of $25-50 \mathrm{mg}$ triamcinolone or $40-80 \mathrm{mg}$ methylprednisolone in a solution containing lidocaine at or near the interspace corresponding to the distribution of pain. Repetitive lumbar ESI have increased 
efficacy. It is hypothesised that it could be in part related to repetitive steroid uptake from epidural veins in postepidural space as well as from blood vessels in subarachnoid space after steroid passive diffusion across the dura.

Salimzadeh et al. designed a study for comparison of two doses of methylprednisolone and they found that in the case of lumbar radicular pain, ESI with low dose $(40 \mathrm{mg})$ methylprednisolone is as effective as high dose $(80 \mathrm{mg})$ with comparable results [50].

The effects of epidurally administered corticosteroids stem from their ability to inhibit the synthesis of prostaglandins, their anti-inflammatory effects, and their ability to inhibit ectopic discharges from injured sensory nerves [51]. Local anesthetics exert their analgesic effects by blocking the conduction in nerves via their effects on $\mathrm{Na}^{+}$channels and suppressing the ectopic signal generation in injured nerves. In addition to providing temporary pain relief, local anesthetics may provide prolonged benefits by putatively interrupting the cycle of pain. Although it seems logical that a larger dose of steroids injected around the affected nerve root would provide more effective analgesia than a smaller dose, the ideal dose and type of steroid has yet to be determined.

Positive results from epidural steroids vary from $20 \%$ to 95\% and may depend on route of injection. Lumbar ESI can be accomplished by one of three methods: caudal (C), interlaminar (IL), or transforaminal (TF). Each technique has been reported to be effective for reducing lower extremity radicular pain. The TF route of epidural steroid placement is more effective than the $\mathrm{C}$ or IL routes. The potential benefits of a transforaminal approach may include minimal risk for dural puncture, better delivery of medication to the site of pathology, increased spread into the ventral epidural space, and subsequently a reduced amount of medication necessary to produce the desired effect.

Kay et al. in their study observed that $80 \mathrm{mg}$ triamcinolone lumbar ESI results in acute suppression of HPA axis ( $\downarrow$ symbol means decreased plasma ACTH and $\downarrow$ symbol means decreased plasma cortisol) 15 minute after injection. Median suppresion of HPA axis was $<1$ month and all patients recovered by 3 months. So, proper perioperative steroid replacement is required if patient undergoes any stress/illness/surgery [52].

\section{Usage of Steroids in Traumatic Spinal Cord Injury}

The use of steroids remains controversial for cord injuries because improvement is minimal and difficult to document. A suggested protocol for traumatic cord injury includes the use of high dose methyl prednisolone with an IV bolus of $30 \mathrm{mg} / \mathrm{Kg}$ followed by $5.4 \mathrm{mg} / \mathrm{kg} / \mathrm{hr}$ infusion for $23 \mathrm{hrs}$. In case of injury to spinal cord, steroids have to be used within $8 \mathrm{hrs}$ for any benefit [53]. Some of the partial cord syndromes have been reported to respond favorably and prompted the maintenance of steroids through subacute interval of one week followed by weaning.

Harish et al. [54] has reported the case of 23-yearold man who was admitted to a community hospital after a diving accident. A workup showed only a $C_{5}$ burst fracture with a $C_{4-5}$ sensory (to pinprick) and motor level (intact diaphragmatic function, paresis below elbow flexors). The bolus dose of dexamethasone $560 \mathrm{mg}$ was followed by an infusion at $100 \mathrm{mg} / \mathrm{hr}$.

Approximately $6 \mathrm{~h}$ postinjury, his dexamethasone infusion was changed to methylprednisolone $5.4 \mathrm{mg} / \mathrm{kg} / \mathrm{hr}$ Surgery was done. Postoperatively, he was transferred to the intensive care unit where his methylprednisolone infusion was discontinued after a $23 \mathrm{hr}$ total infusion time as per the NASCIS (National Acute Spinal Cord Injury Study) protocol.

This patient developed adrenal insufficiency (AI) after this protocol. Although a definitive causal relationship between the steroids and AI was not established, their temporal association and the exclusion of other possible etiologies led them to postulate that AI was a complication of the steroid protocol. Clinicians should, therefore, consider AI in patients with spinal cord injury receiving glucocorticoids, a population in whom it may otherwise go undiagnosed and untreated.

\section{Steroids in Hyperreactive Airway}

Steroids by virtue of their anti-inflammatory action lead to decreased mucosal oedema and prevention of release of bronchoconstricting substances. They are also said to have a permissive role for bronchodilator medication, that is, they enhance the efficacy of bronchodilator. They are useful in both acute as well as chronic hyperreactive diseases. For this purpose they can be administered orally, parenterally, or in aerosol form. The most commonly encountered hyper reactive states in anaesthetic practice are patients with history of asthma, recent upper respiratory tract infection, difficult airway, multiple intubation attempts, aspiration, foreign body bronchus, airway surgeries, and COPD.

\section{Aspiration Pneumonitis}

The use of steroids in treatment of aspiration pneumonitis is controversial. There is evidence in animals that steroids administered immediately after the inhalation of acidic gastric content may be effective in decreasing pulmonary damage. Conversely, other data show no beneficial effect or suggest that the use of steroids may enhance the likelihood of gram negative pneumonia. Despite the absence of confirming evidence that steroids are beneficial, it is not uncommon for the treatment of aspiration pneumonitis to include the emperical use of pharmacologic doses of these drugs.

\section{Post Intubation Laryngeal Oedema}

Steroids are commonly given after multiple attempts at intubation to preventpost operative laryngeal oedema. Steroids by virtue of their anti-inflammatory action lead to decreased mucosal oedema. Dexamethasone $0.1-0.2 \mathrm{mg} / \mathrm{kg}$ iv is commonly used, though its efficacy in treatment of this condition has not been confirmed. Dexamethasone $0.6 \mathrm{mg} / \mathrm{kg}$ orally is effective treatment for children with mild croup. 


\section{Sepsis and Steroid}

Septic shock has a crude mortality rate of 45\% [55] and claims the lives of 90000 people each year in the USA alone. Some recent approaches have shown promise in prevention or treatment of sepsis and septic shock. They include tight glycaemic control, early haemodynamic goaldirected therapy, infusion of activated protein $\mathrm{C}$, and use of corticosteroids.

Patients having severe sepsis or in septic shock were found to have occult or unrecognized adrenal insufficiency; incidence may be as high as $28 \%$ in seriously ill patients. Clinically, it has been shown that in sepsis with adrenal insufficiency, steroid supplementation was associated with significantly higher rate of success in withdrawal of vasopressor therapy. Corticosteroids have a long history of use in intensive care for septic shock and were extensively used in high dose for a short duration until the mid 1980s when large multicentre trials showed they were of no benefit. This finding was confirmed in subsequent meta-analyses [56]. Their use for septic shock was largely abandoned.

However, since then, small explanatory trials of physiological "stress" doses of corticosteroids have demonstrated a reduced need for vasopressor drugs to maintain cardiovascular homeostasis, a possible surrogate for improved clinical outcomes [57]. The benefits of steroids may result from suppression of overexuberant and dysregulated immune responses, suppression of inflammatory responses through a variety of mechanisms, and upregulation of adrenoreceptor function. However, adverse sequelae are well described and effects of corticosteroids on development of nosocomial infection, reactivation of latent infection, hyperglycaemia, bone metabolism, and psychosis as well as intensive care associated paresis must also be considered. Thus, surrogate measures describing benefit may be misleading, and adequately designed and powered clinical outcome studies are essential to assure that potential benefits outweigh known adverse effects. Given the apparently large survival benefit, as well as the ease of administration and low costs of corticosteroid treatment, these findings could assume great importance for the $77 \%$ of patients with insufficient adrenal reserve as defined by their response to corticotropin. Several recent reviews and commentaries have since highlighted these beneficial results [58].

For patients who have a critical illness such as septic shock, Coursin and Wood [59] have recommended 50$100 \mathrm{mg}$ of hydrocortisone every $6-8 \mathrm{hrs}$ or $0.18 \mathrm{mg} / \mathrm{kg} / \mathrm{hr}$ as a continuous infusion, together with fludrocortisone $0.05 \mathrm{mg}$ daily. The current evidence does not support the use of hydrocortisone doses above $200 \mathrm{mg}$ /day. Arafah [60] reported that after intravenous boluses of hydrocortisone $50 \mathrm{mg}$ had been given every 6 hours, peak plasma cortisol levels were over $100 \mu \mathrm{g} / \mathrm{dL}(2760 \mathrm{nmol} / \mathrm{L})$ and nadir levels remained elevated at 40-50 $\mu \mathrm{g} / \mathrm{dL}(1100-1380 \mathrm{nmol} / \mathrm{L})$ [61]. Ho et al. [62] showed that, during continuous hydrocortisone infusion $(10 \mathrm{mg} / \mathrm{hr})$, plasma total cortisol levels were over $3000 \mathrm{nmol} / \mathrm{L}$, well above the levels reported in patients with septic shock (mean $880 \mathrm{nmol} / \mathrm{L}$ ) [63]. Another study found that the majority of cortisol levels were between 552 and $1242 \mathrm{nmol} / \mathrm{L}$ in intensive care unit patients with severe sepsis or septic shock. While it is evident that the glucocorticoid dose should not exceed $200 \mathrm{mg}$ /day, the optimal dose for managing septic shock in patients with adrenal insufficiency has not been evaluated in controlled clinical trials. Mineralocorticoid supplementation with fludrocortisone is not required in patients with secondary adrenal insufficiency or in those with primary adrenal insufficiency receiving more than $50 \mathrm{mg}$ hydrocortisone daily, given its potent mineralocorticoid activity at high doses [61].

\section{Steroids and Post Extubation Stridor}

Postextubation stridor typically occurs in people who have been intubated for several days, with a prevalence of up to $37 \%$ of intubated patients in the intensive care unit [64]. As many as $80 \%$ of patients who develop the complication require reintubation prolonging their stay in the ICU and increasing their risk for complications, such as nosocomial pneumonia. Some practitioners use a cuff-leak test in adults to decide whether to use steroids before extubation [64]. If there is a small difference between exhaled tidal volume with the cuff inflated versus deflated, inflammation is likely present. In these cases, physicians often use steroids to reduce inflammation before the endotracheal tube is removed. But because steroids have adverse effects, including hypertension and hyperglycemia, they should be avoided unless necessary.

Farrell et al. [65], found a statistically significant reduction of reintubation for any reason across all age groups, including neonatal and padiatric populations. The effect of steroids was more pronounced if they were administered at least $12 \mathrm{hrs}$ prior to extubation for patients who have been intubated for more than 3 days or who are at increased risk of reintubation. The analysis also found a reduction of laryngeal oedema in participants receiving the steroids.

Fan et al. [66], in their study, found that multiple doses of steroids reduce the risk for edema and reintubation, whereas a single dose only shows a non significant trend toward effectiveness.

Jaber et al. [67] concluded that steroids were most useful when administered in high risk patients, as determined by a cuff-leak test, and when the steroids were administered at least $4 \mathrm{hrs}$ before extubation. Benefits were less clear if patients were not selected according to risk.

\section{Steroid and Anaphylaxis}

Anesthesiologists use a myriad of drugs during the provision of an anesthetic. Many of these drugs have side effects that are dose-related, and some lead to severe immune-mediated adverse reactions. Anaphylaxis is the most severe immunemediated reaction; it generally occurs on reexposure to a specific antigen and requires the release of proinflammatory mediators. Symptoms may include all organ systems and present with bronchospasm and cardiovascular collapse in the most severe cases. Management of anaphylaxis includes discontinuation of the presumptive drug (or latex) and anesthetic, aggressive pulmonary and cardiovascular support, and epinephrine. The incidence of anaphylaxis during 
anesthesia is very difficult to estimate but has been calculated to range from 1 in 3,500 [68].

A survey of anaphylaxis during anesthesia demonstrated that cardiovascular symptoms $(73.6 \%)$, cutaneous symptoms $(69.6 \%)$, and bronchospasm $(44.2 \%)$ were the most common clinical features. Muscle relaxants are associated with the most frequent incidence of anaphylaxis, and over the last two decades, natural rubber latex (NRL, or cis-1,4polyisoprene) has emerged as the second most common cause of anaphylaxis.

Corticosteroids can decrease the airway swelling and prevent recurrence of symptoms, as seen in biphasic or protracted anaphylaxis. Hydrocortisone is the preferred steroid because it has a fast onset of action. Glucocorticoids can supplement primary therapy to suppress manifestations of allergic diseases of limited duration like hay fever, serum sickness, urticaria, contact dermatitis, drug reactions, bee stings, and angioneurotic oedema. In mild forms of allergy, antihistamines are the first choice of drugs. In very severe forms, iv methylprednisolone $125 \mathrm{mg}$, every 6 hours or equivalent dose, can be used to obtain relief.

\section{Application of Steroids in Cerebral Oedema}

Steroids are of value in reduction or prevention of cerebral edema associated with parasitic infections or neoplasms [69]. The mechanism by which steroids influence vasogenic edema are thought to include one or more of the following.

(i) Stabilisation of cerebral endothelium, leading to decrease in plasma filtration.

(ii) Increase in lysosomal activity of cerebral capillaries.

(iii) Inhibition of release of potentially toxic substances such as free radicals, fatty acids, and prostaglandins.

(iv) Electrolyte shifts favoring transcapillary efflux of fluid.

(v) Increase of local and global cerebral glucose use, leading to improved neuronal function.

In the management of patient with malignant brain tumor, it is not uncommon for subjects who are somnolent or stuporous on admission to respond within hours to loading dose of dexamethasone (8-32 mg) and appear alert and without neurological deficits by the following day [69].

Controversy exists regarding response to steroid use in closed head injury. As prolonged steroids are associated with various side effects, among them hyperglycemia and increased predisposition to infection which are harmful in head injury patient. So, use of steroids in traumatic brain injury is considered obsolete.

\section{Efficacy of Steroids in Cardiac Arrest}

Cardiac arrest causes global cerebral ischemia resulting in intracellular cytotoxic edema. Dexamethasone $(0.5-1 \mathrm{mg} / \mathrm{kg})$ is given initially followed by $4-8 \mathrm{mg}$ every $6 \mathrm{hrs}$ but methylprednisolone may be as effective [70]. The efficacy of steroids in treatment of perifocal vasogenic oedema as occurs in intrinsic mass lesions is well established but its value in intracellular cytotoxic edema is still unproved. Indeed steroids have not been shown to improve survival or neurologic recovery rate after arrest and there administration is not recommended.

\section{Adverse Drug Effects of Steroid Supplementation}

19.1. Risks with Short-Term (Perioperative) Supplementation. Aggravation of hypertension, fluid retention, stress ulcers, GI bleeding, silent perforation, psychiatric disturbances, delayed and abnormal wound healing, hypokalemia, osteoporosis, increased susceptibility to infection, and decreased glucose tolerance.

19.2. Risks with Long-Term Supplementation. HPA axis suppression, hypokalemia metabolic alkalosis, oedema, weight gain, hyperglycemia, redistribution of body fat, buffalo hump, and proximal skeletal muscle myopathy.

\section{Conclusion}

As discussed in the paper, there is an increasing application of steroid therapy during perioperative period for various purposes. Because they exert effects on almost every organ system, the clinical use of and withdrawal from corticosteroids are complicated by a number of serious side effects, some of which are life-threatening. Therefore, the decision to institute therapy with corticosteroids always requires careful consideration of the relative risks and benefits in each patient. With improved medical and diagnostic facilities, it is more likely for any anaesthesiologist to encounter patients on long/ short-term steroid therapy. Patients on long-term steroids are a challenge to any anaesthesiologist. It is incumbent on us to become familiar with all aspects of steroid physiology, functions, and adverse effects to avoid potentially life threatening situations in the perioperative period.

\section{References}

[1] L. L. Bruton, J. S. Lazo, and K. L. Parker, Goodman \& Gilman'S the Pharmacological Basis of Therapeutics, 11th edition, 2006.

[2] Text book of Human Anatomy by Gray's, 68th edition.

[3] Textbook of Physiology by Guyton \& Hall, 2nd edition.

[4] Review of Medical Physiology by William F. Ganong, 24th edition.

[5] Braunwald et al., Harrison's Principles of Internal Medicine by Kasper, 17th edition.

[6] C. Jung and W. J. Inder, "Management of adrenal insufficiency during the stress of medical illness and surgery," Medical Journal of Australia, vol. 188, no. 7, pp. 409-413, 2008.

[7] Essentials of Medical Pharmacology by KD Tripathi, 6th edition.

[8] R. A. Donald, E. G. Perry, G. A. Wittert et al., "The plasma $\mathrm{ACTH}, \mathrm{AVP}, \mathrm{CRH}$ and catecholamine responses to conventional and laparoscopic cholecystectomy," Clinical Endocrinology, vol. 38, no. 6, pp. 609-615, 1993.

[9] I. E. Widmer, J. J. Puder, C. König et al., "Cortisol response in relation to the severity of stress and illness," Journal of Clinical 
Endocrinology and Metabolism, vol. 90, no. 8, pp. 4579-4586, 2005.

[10] R. Udelsman, J. A. Norton, and S. E. Jelenich, "Responses of the hypothalamic-pituitary-adrenal and renin-angiotensin axes and the sympathetic system during controlled surgical and anesthetic stress," Journal of Clinical Endocrinology and Metabolism, vol. 64, no. 5, pp. 986-994, 1987.

[11] B. M. Arafah, "Review: hypothalamic pituitary adrenal function during critical illness: limitations of current assessment methods," Journal of Clinical Endocrinology and Metabolism, vol. 91, no. 10, pp. 3725-3745, 2006.

[12] L. Wise, H. W. Margraf, and W. F. Ballinger, "A new concept on the pre- and postoperative regulation of cortisol secretion," Surgery, vol. 72, no. 2, pp. 290-299, 1972.

[13] B. M. Arafah, S. H. Kailani, K. E. Nekl, R. S. Gold, and W. R. Selman, "Immediate recovery of pituitary function after transsphenoidal resection of pituitary macroadenomas," Journal of Clinical Endocrinology and Metabolism, vol. 79, no. 2, pp. 348-354, 1994.

[14] A. Crown and S. Lightman, "Why is the management of glucocorticoid deficiency still controversial: a review of the literature," Clinical Endocrinology, vol. 63, no. 5, pp. 483-492, 2005.

[15] S. A. Jabbour, "Steroids and the surgical patient," Medical Clinics of North America, vol. 85, no. 5, pp. 1311-1317, 2001.

[16] F. H. De Jong, C. Mallios, and C. Jansen, "Etomidate suppresses adrenocortical function by inhibition of $11 \beta$-hydroxylation," Journal of Clinical Endocrinology and Metabolism, vol. 59, no. 6, pp. 1143-1147, 1984.

[17] L. D. VANDAM and F. D. MOORE, "Adrenocortical mechanisms related to anesthesia," Anesthesiology, vol. 21, pp. 531$552,1960$.

[18] Anaesthesia Co Existing Disease by Stoelting, 5th edition.

[19] B. Chernow, H. R. Alexander, and R. C. Smallridge, "Hormonal responses to graded surgical stress," Archives of Internal Medicine, vol. 147, no. 7, pp. 1273-1280, 1987.

[20] T. A. Howlett, "An assessment of optimal hydrocortisone replacement therapy," Clinical Endocrinology, vol. 46, no. 3, pp. 263-268, 1997.

[21] W. Oelkers, "Current concepts: adrenal insufficiency," The New England Journal of Medicine, vol. 335, no. 16, pp. 12061212, 1996.

[22] A. S. Krasner, "Glucocorticoid-induced adrenal insufficiency," Journal of the American Medical Association, vol. 282, no. 7, pp. 671-676, 1999.

[23] P. M. Stewart, "The adrenal cortex," in Williams Textbook of Endocrinology, P. R. Larsen, H. M. Kronenberg, S. Melmed, and K. S. Polonsky, Eds., pp. 491-551, Saunders, Philadelphia, $\mathrm{Pa}$, USA, 10th edition, 2003.

[24] G. E. La Rochelle Jr., A. G. La Rochelle, R. E. Ratner, and D. G. Borenstein, "Recovery of the hypothalamic-pituitary-adrenal (HPA) axis in patients with rheumatic diseases receiving lowdose prednisone," American Journal of Medicine, vol. 95, no. 3, pp. 258-264, 1993.

[25] R. Schlaghecke, E. Kornely, R. T. Santen, and P. Ridderskamp, "The effect of long-term glucocorticoid therapy on pituitaryadrenal responses to exogenous corticotropin-releasing hormone," The New England Journal of Medicine, vol. 326, no. 4, pp. 226-230, 1991.

[26] R. A. Donald, E. G. Perry, G. A. Wittert et al., "The plasma $\mathrm{ACTH}, \mathrm{AVP}, \mathrm{CRH}$ and catecholamine responses to conventional and laparoscopic cholecystectomy," Clinical Endocrinology, vol. 38, no. 6, pp. 609-615, 1993.
[27] G. Nicholson, J. M. Burrin, and G. M. Hall, "Peri-operative steroid supplementation," Anaesthesia, vol. 53, no. 11, pp. 1091-1104, 1998.

[28] R. Udelsman, D. S. Goldstein, D. L. Loriaux, and G. P. Chrousos, "Catecholamine-glucocorticoid interactions during surgical stress," Journal of Surgical Research, vol. 43, no. 6, pp. 539-545, 1987.

[29] H. Kehlet, "A rational approach to dosage and preparation of parenteral glucocorticoid substitution therapy during surgical procedures: a short review," Acta Anaesthesiologica Scandinavica, vol. 19, no. 4, pp. 260-264, 1975.

[30] M. Salem, R. E. Tainsh, J. Bromberg, D. L. Loriaux, and B. Chernow, "Perioperative glucocorticoid coverage: a reassessment 42 years after emergence of a problem," Annals of Surgery, vol. 219, no. 4, pp. 416-425, 1994.

[31] L. K. Poulson, "Rational substitution therapy for steroid traeted patients," Anaesthesia, vol. 33, pp. 59-60, 1978.

[32] William Textbook of Endocrinology, Wilson \& Foster, 8th edition.

[33] H. Kehlet and C. Binder, "Alterations in distribution volume and biological half-life of cortisol during major surgery," Journal of Clinical Endocrinology and Metabolism, vol. 36, no. 2, pp. 330-333, 1973.

[34] P. H. Tan, K. Liu, C. H. Peng, L. C. Yang, C. R. Lin, and C. Y. Lu, "The effect of dexamethasone on postoperative pain emesis after intrathecal neostigmine," Anesthesia and Analgesia, vol. 92 , no. 1, pp. 228-232, 2001.

[35] B. R. Baxendale, M. Vater, and K. M. Lavery, "Dexamethasone reduces pain and swelling following extraction of third molar teeth," Anaesthesia, vol. 48, no. 11, pp. 961-964, 1993.

[36] M. S. Aapro and D. S. Alberts, "Dexamethasone as an antiemetic in patients treated with cisplatin," The New England Journal of Medicine, vol. 305, no. 9, p. 520, 1981.

[37] W. M. Splinter and E. J. Rhine, "Low-dose ondansetron with dexamethasone more effectively decreases vomiting after strabismus surgery in children than does high-dose ondansetron," Anesthesiology, vol. 88, no. 1, pp. 72-75, 1998.

[38] F. I. Catlin and W. J. Grimes, "The effect of steroid therapy on recovery from tonsillectomy in children," Archives of Otolaryngology, vol. 117, no. 6, pp. 649-652, 1991.

[39] T. J. Hursti, M. Fredrikson, G. Steineck, S. Borjeson, C. J. Furst, and C. Peterson, "Endogenous cortisol exerts antiemetic effect similar to that of exogenous corticosteroids," British Journal of Cancer, vol. 68, no. 1, pp. 112-114, 1993.

[40] T. J. Gan, T. Meyer, C. C. Apfel et al., "Consensus guidelines for managing postoperative nausea and vomiting," Anesthesia and Analgesia, vol. 97, no. 1, pp. 62-71, 2003.

[41] A. Gupta, C. L. Wu, N. Elkassabany, C. E. Krug, S. D. Parker, and L. A. Fleisher, "Does the routine prophylactic use of antiemetics affect the incidence of postdischarge nausea and vomiting following ambulatory surgery? A systematic review of randomized controlled trials," Anesthesiology, vol. 99, no. 2, pp. 488-495, 2003.

[42] B. R. Baxendale, M. Vater, and K. M. Lavery, "Dexamethasone reduces pain and swelling following extraction of third molar teeth," Anaesthesia, vol. 48, no. 11, pp. 961-964, 1993.

[43] The Italian Group for Antiemetic Research, "Dexamethasone alone or in combination with ondansetron for the prevention of delayed nausea and vomiting induced by chemotherapy," The New England Journal of Medicine, vol. 342, no. 21, pp. 1554-1559, 2000.

[44] V. Aasboe, J. C. Raeder, and B. Groegaard, "Betamethasone reduces postoperative pain and nausea after ambulatory 
surgery," Anesthesia and Analgesia, vol. 87, no. 2, pp. 319-323, 1998.

[45] S. C. Ahlgren, J. F. Wang, and J. D. Levine, "C-fiber mechanical stimulus-response functions are different in inflammatory versus neuropathic hyperalgesia in the rat," Neuroscience, vol. 76, no. 1, pp. 285-290, 1996.

[46] H. Mirzai, I. Tekin, and H. Alincak, "Perioperative use of corticosteroid and bupivacaine combination in lumbar disc surgery: a randomized controlled trial," Spine, vol. 27, no. 4, pp. 343-346, 2002.

[47] A. Movafegh, M. Razazian, F. Hajimaohamadi, and A. Meysamie, "Dexamethasone added to lidocaine prolongs axillary brachial plexus blockade," Anesthesia and Analgesia, vol. 102, no. 1, pp. 263-267, 2006.

[48] K. McCormack, "The spinal actions of nonsteroidal antiinflammatory drugs and the dissociation between their antiinflammatory and analgesic effects," Drugs, vol. 47, no. 5, pp. 28-45, 1994.

[49] Anaesthesia Textbook by Miller, 7th edition.

[50] A. Salimzadeh, G. Alishiri, A. Haghighi, and M. B. Owlia, "Comparison of two doses of corticosteroid in epidural steroid injection for lumbar radicular pain," Singapore Medical Journal, vol. 48, no. 3, pp. 241-245, 2007.

[51] F. Hirata, E. Schiffmann, and K. Venkatasubramanian, "A phospholipase A2 inhibitory protein in rabbit neutrophils induced by glucocorticoids," Proceedings of the National Academy of Sciences of the United States of America, vol. 77, no. 5, pp. 2533-2536, 1980.

[52] J. Kay, J. W. Findling, and H. Raff, "Epidural triamcinolone suppresses the pituitary-adrenal axis in human subjects," Anesthesia and Analgesia, vol. 79, no. 3, pp. 501-505, 1994.

[53] Textbook of Anaesthesia-Pharmaco-Physiology by Stoelting, 4th edition.

[54] Harish et al., "A case report on development of adrenal insufficiency after NASCIS protocol," Anesthesia \& Analgesia, vol. 102, no. 6, pp. 1361-1372, 2006.

[55] R. Bloomfield and D. W. Noble, "Corticosteroids for septic shock-a standard of care?" British Journal of Anaesthesia, vol. 93, no. 2, pp. 178-180, 2004.

[56] R. Lefering and E. A. M. Neugebauer, "Steroid controversy in sepsis and septic shock: a meta-analysis," Critical Care Medicine, vol. 23, no. 7, pp. 1294-1303, 1995.

[57] D. Annane, E. Bellissant, V. Sebille et al., "Impaired pressor sensitivity to noradrenaline in septic shock patients with and without impaired adrenal function reserve," British Journal of Clinical Pharmacology, vol. 46, no. 6, pp. 589-597, 1998.

[58] M. S. Cooper and P. M. Stewart, "Corticosteroid insufficiency in acutely ill patients," The New England Journal of Medicine, vol. 348, no. 8, pp. 727-734, 2003.

[59] D. B. Coursin and K. E. Wood, "Corticosteroid supplementation for adrenal insufficiency," Journal of the American Medical Association, vol. 287, no. 2, pp. 236-240, 2002.

[60] B. M. Arafah, "Review: hypothalamic pituitary adrenal function during critical illness: limitations of current assessment methods," Journal of Clinical Endocrinology and Metabolism, vol. 91, no. 10, pp. 3725-3745, 2006.

[61] F. M. Kenny, C. Preeyasombat, and C. J. Migeon, "Cortisol production rate. II. Normal infants, children, and adults," Pediatrics, vol. 37, no. 1, pp. 34-42, 1966.

[62] J. T. Ho, H. Al-Musalhi, M. J. Chapman et al., "Septic shock and sepsis: a comparison of total and free plasma cortisol levels," Journal of Clinical Endocrinology and Metabolism, vol. 91, no. 1, pp. 105-114, 2006.
[63] A. H. Hamrahian, T. S. Oseni, and B. M. Arafah, "Measurements of serum free cortisol in critically Ill patients," The New England Journal of Medicine, vol. 350, no. 16, pp. 1629-1638, 2004.

[64] D. Annane, V. Sébille, G. Troché, J. C. Raphaël, P. Gajdos, and E. Bellissant, "A 3-level prognostic classification in septic shock based on cortisol levels and cortisol response to corticotropin," Journal of the American Medical Association, vol. 283, no. 8, pp. 1038-1045, 2000.

[65] C. Farrell, J. McCaffrey, P. Whiting, A. Dan, S. M. Bagshaw, and A. P. Delaney, "Corticosteroids to prevent extubation failure: a systematic review and meta-analysis," Intensive Care Medicine, vol. 35, no. 6, pp. 977-986, 2009.

[66] T. Fan, G. Wang, B. Mao et al., "Prophylactic administration of parenteral steroids for preventing airway complications after extubation in adults: meta-analysis of randomised placebo controlled trials," British Medical Journal, vol. 337, Article ID a1841, 2008.

[67] S. Jaber, B. Jung, G. Chanques, F. Bonnet, and E. Marret, "Effects of steroids on reintubation and post-extubation stridor in adults: meta-analysis of randomised controlled trials," Critical Care, vol. 13, no. 2, article R49, 2009.

[68] D. L. Hepner and M. C. Castells, "Anaphylaxis during the perioperative period," Anesthesia and Analgesia, vol. 97, no. 5, pp. 1381-1395, 2003.

[69] V. K. Grover, R. Babu, and S. P. S. Bedi, "Steroid therapycurrent indications in practice," International Jugglers' Association, vol. 51, no. 5, pp. 389-339, 2007.

[70] M. C. Laxenaire and P. M. Mertes, "Anaphylaxis during anaesthesia. Results of a two-year survey in France," British Journal of Anaesthesia, vol. 87, no. 4, pp. 549-558, 2001. 


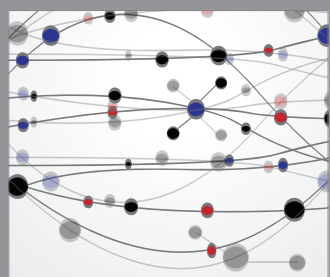

The Scientific World Journal
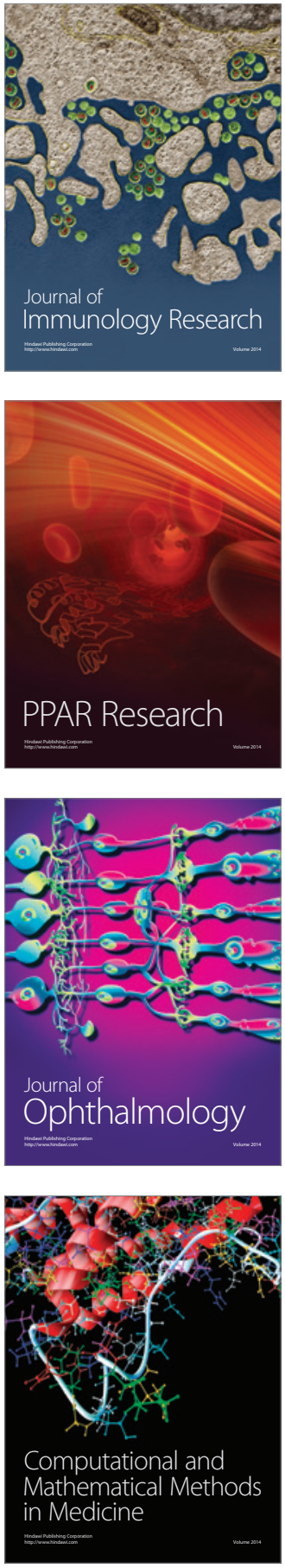



Gastroenterology

Research and Practice
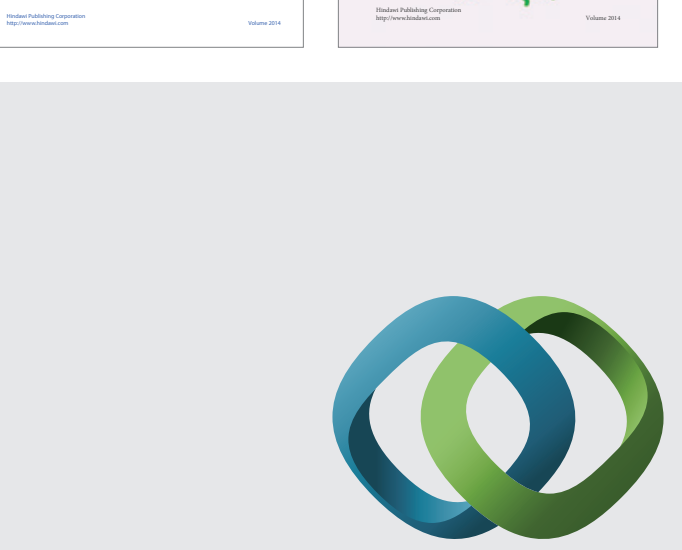

\section{Hindawi}

Submit your manuscripts at

http://www.hindawi.com
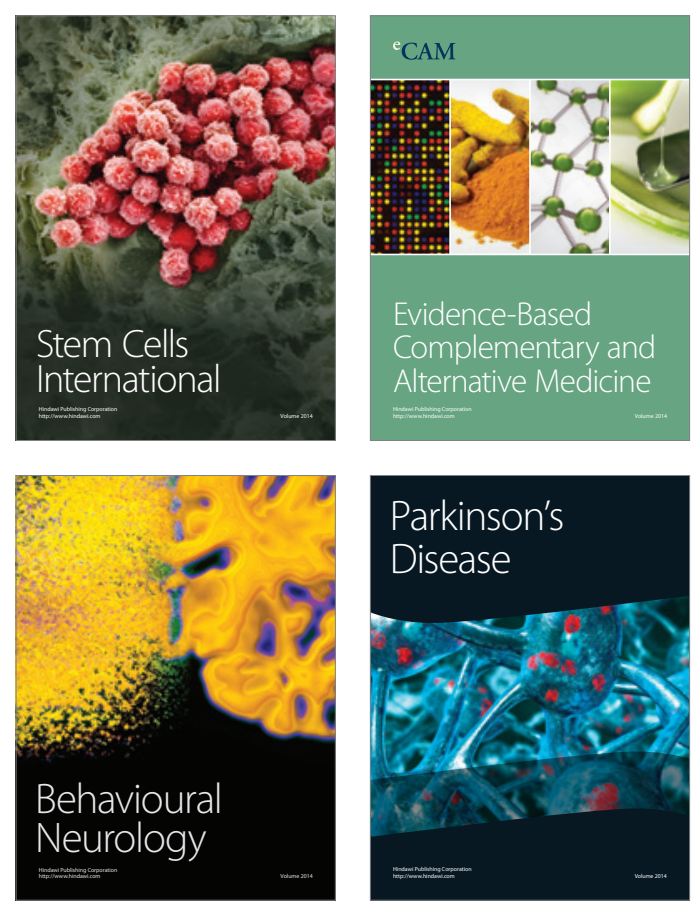

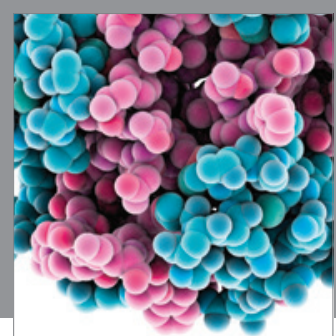

Journal of
Diabetes Research

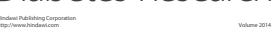

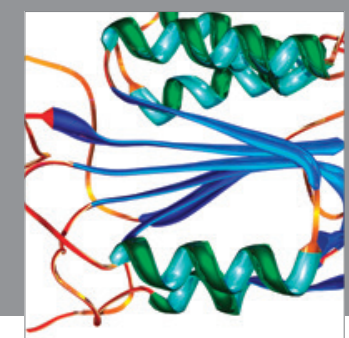

Disease Markers
